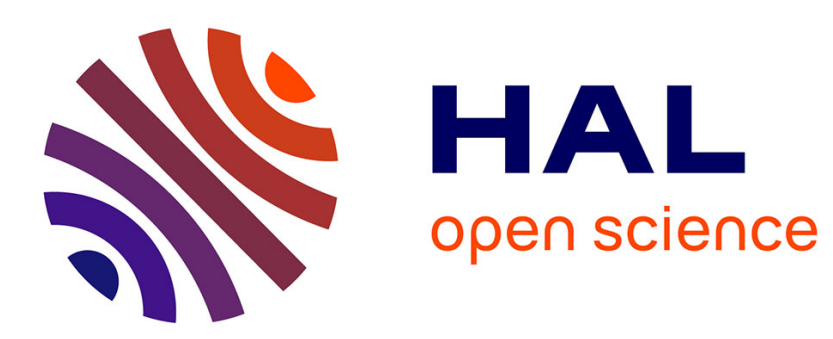

\title{
Ultrasonic rheology of visco-elastic materials using shear and longitudinal waves
}

\author{
Gautier Lefebvre, Régis Wunenburger, Tony Valier-Brasier
}

\section{To cite this version:}

Gautier Lefebvre, Régis Wunenburger, Tony Valier-Brasier. Ultrasonic rheology of visco-elastic materials using shear and longitudinal waves. Applied Physics Letters, 2018, 112 (24), 10.1063/1.5029905 . hal-01921803

\section{HAL Id: hal-01921803 \\ https://hal.sorbonne-universite.fr/hal-01921803}

Submitted on 14 Nov 2018

HAL is a multi-disciplinary open access archive for the deposit and dissemination of scientific research documents, whether they are published or not. The documents may come from teaching and research institutions in France or abroad, or from public or private research centers.
L'archive ouverte pluridisciplinaire HAL, est destinée au dépôt et à la diffusion de documents scientifiques de niveau recherche, publiés ou non, émanant des établissements d'enseignement et de recherche français ou étrangers, des laboratoires publics ou privés. 


\title{
Ultrasonic rheology of visco-elastic materials using shear and longitudinal waves
}

\author{
Gautier Lefebvre, Régis Wunenburger, and Tony Valier-Brasier \\ Sorbonne Université, Centre National de la Recherche Scientifique, UMR 7190, Institut Jean Le Rond \\ O'Alembert, F-75005 Paris, France
}

(Received 16 March 2018; accepted 6 June 2018; published online 15 June 2018)

\begin{abstract}
We present a technique for determining the rheology of visco-elastic materials in the ultrasonic frequency range based on the measurement of the longitudinal and shear waves reflected and transmitted by a slab of material with a controlled thickness sandwiched between two delay lines. The high sensitivity of this Fabry-Pérot resonator-based technique allows one to access characteristic relaxation times associated with visco-elasticity. As a useful application of the technique, we characterize the Olympus SWC-2 shear-wave couplant in the $0.7-5 \mathrm{MHz}$ frequency range. Published by AIP Publishing. https://doi.org/10.1063/1.5029905
\end{abstract}

A thorough determination of the rheological properties of visco-elastic materials at ultrasonic frequencies, namely, both their complex longitudinal $(M)$ and shear $(G)$ moduli, requires the analysis of the propagation of both longitudinal and shear waves. While several techniques allow the determination of $M$ using longitudinal (L) waves, only a few address the determination of $G$ using shear (T) waves.

Concerning $\mathrm{L}$ waves, apart from immersion techniques, pioneering metrological achievements by Papadakis involved a slab of material bonded to a buffer rod playing the role of a delay line. ${ }^{1-3}$ The $\mathrm{L}$ impedance and attenuation of the material were deduced from the analysis of the echoes due to the reflections of an $\mathrm{L}$ pulse by the line-slab and slabair interfaces. Later, several authors have used the same kind of technique to determine the mass density of liquids. ${ }^{4-6}$ Recently, a line-slab-line system was implemented to accurately determine the acoustic attenuation in a liquid layer from the analysis of the reflection of L pulses. ${ }^{7}$ This technique has been used later to monitor the polymerization of an epoxy layer from the determination of $\mathrm{L}$ velocity and attenuation in epoxy. ${ }^{8}$ Concerning shear waves, the classical technique based on the conversion to a shear wave of an $\mathrm{L}$ wave incident on an immersed slab beyond the critical angle has been applied to visco-elastic materials. ${ }^{9}$ Longin et al. have determined the shear modulus of visco-elastic materials by analyzing the reflection at grazing incidence of a $\mathrm{T}$ pulse by the line-material interface. ${ }^{10}$ All these techniques are based on the separation of echoes and hence require thick samples compared to wavelength, and most of them assume weak attenuation.

We present here a technique involving both $\mathrm{L}$ and $\mathrm{T}$ waves which allows one to completely determine the frequency-dependent rheological properties of visco-elastic materials. The technique involves a line-slab-line setup and allows one to determine the complex moduli $M$ and $G$ of the visco-elastic material constituting the slab from the analysis of the reflection and transmission of $\mathrm{L}$ or $\mathrm{T}$ pulses by the slab at normal incidence. The use of a Fabry-Pérot resonator model to describe the reflection and transmission by the slab allows one to handle thin slabs, to study highly absorbing materials, to consider low frequencies, and to address dispersion over broad frequency bands. Such a model has already been used to characterize the visco-elastic properties of solid samples using torsional waves. ${ }^{11}$

The propagation of $\mathrm{L}$ or $\mathrm{T}$ waves through a line-slabline system is modeled as a one-dimensional propagation of plane waves through a three-layer system composed of two half-spaces made of the same material and a layer with thickness $h$ made of the material to be characterized. We consider an incident $\mathrm{L}$ or $\mathrm{T}$ pulse lasting possibly longer than the round trip time-of-flight in the layer. Consequently, the effect of multiple reflections inside the layer has to be taken into account. The spectral stress-reflection and transmission coefficients $\mathcal{R}$ and $\mathcal{T}$ of the three-layer system to be considered are thus given by ${ }^{12}$

$$
\left\{\begin{array}{l}
\mathcal{R}=\frac{j\left(Z^{2}-Z_{\mathrm{d}}^{2}\right) \sin (k h)}{j\left(Z_{\mathrm{d}}^{2}+Z^{2}\right) \sin (k h)+2 Z_{\mathrm{d}} Z \cos (k h)}, \\
\mathcal{T}=\frac{2 Z_{\mathrm{d}} Z}{j\left(Z_{\mathrm{d}}^{2}+Z^{2}\right) \sin (k h)+2 Z_{\mathrm{d}} Z \cos (k h)},
\end{array}\right.
$$

where $j^{2}=-1, k=\omega / c$ is the complex wavenumber at pulsation $\omega$ in the layer with velocity $c, \rho$ (resp. $\rho_{\mathrm{d}}$ ) the mass density of the material constituting the layer (resp. the delay lines), and $Z=\rho c$ and $Z_{\mathrm{d}}=\rho_{\mathrm{d}} c$ their respective impedance. The $\mathrm{L}$ and $\mathrm{T}$ wave velocities in the layer are $c_{\mathrm{L}}=\sqrt{\left(K+\frac{4}{3} G\right) / \rho}$ and $c_{\mathrm{T}}=\sqrt{G / \rho}$, where $K$ and $G$ are the complex bulk and shear moduli of the material constituting the layer. With the expressions of coefficients $\mathcal{R}$ and $\mathcal{T}$ for $\mathrm{L}$ and $\mathrm{T}$ waves involving six elastic parameters, knowing $\rho_{d}, c_{\mathrm{L}}^{\mathrm{d}}, c_{\mathrm{T}}^{\mathrm{d}}$, and $\rho$, the experimental determination of $\mathcal{R}$ and $\mathcal{T}$ allows one to access the frequency-dependence of $G$ and $K$.

We use two identical $H=60 \mathrm{~mm}$ thick parallelepipedic delay lines made of 2017A Dural alloy, whose normalized properties are density $\rho_{\mathrm{d}}=2790 \mathrm{~kg} \mathrm{~m}^{-3}$, Young modulus $E=74 \mathrm{GPa}$, and Poisson's ratio $\nu=0.33$. This corresponds to $\mathrm{L}$ velocity $c_{\mathrm{L}}^{\mathrm{d}}=6269 \mathrm{~m} \mathrm{~s}^{-1}$ and $\mathrm{T}$ velocity $c_{\mathrm{T}}^{\mathrm{d}}=3157 \mathrm{~m} \mathrm{~s}^{-1}$. Their faces were mirror-polished. Two contact transducers are mounted in contact on each delay line using a small amount (7 mg) of Olympus SWC-2 couplant. As sketched in Fig. 1, a 


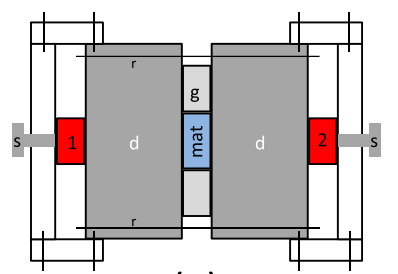

(a)

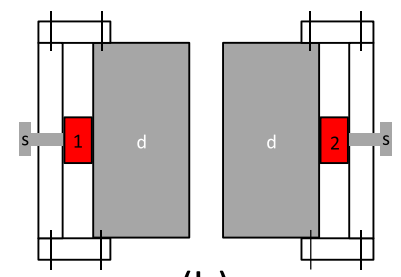

(b)
FIG. 1. Sketch of the two different configurations of the experimental setup. 1 and 2: transducers, d: delay lines, g: spacer, mat: material of interest, s: screw tightening together the transducer and the delay line, and r: threaded rod tightening together the spacer, the material slab, and the delay lines. (a) Mounted setup used to measure the signals reflected and transmitted by the line-slab-line system. (b) Unmounted setup used to measure the calibration signals.

tightening screw applies a constant force on the transducer in order to obtain an efficient transmission of $\mathrm{L}$ and $\mathrm{T}$ waves. The torque applied on these screws is reproducibly set at $2 \mathrm{Nm}$.

In order to control the thickness of the sample of the visco-elastic material, the delay lines are separated by a circular spacer surrounding the material sample and tightened together using three threaded rods, as sketched in Fig. 1(a). The third one is not visible in the sketch as it is in the same plane as the transducers, behind them.

The setup is put in a refrigerated incubator at $(5.0 \pm 0.2)^{\circ} \mathrm{C}$, which importantly ensures a steady transmission by the couplant layer squeezed between the transducer and the delay line after a few hours (at room temperature, this transmission drifts over days). We checked that the $\mathrm{L}$ and $\mathrm{T}$ velocities in the delay lines measured at $5{ }^{\circ} \mathrm{C}$ correspond to the above given properties of Dural at ambient temperature within measurement uncertainties. Two different kinds of contact transducers are used: a pair of $\mathrm{T}$ wave 2.25 MHz Olympus V154.RM transducers and a pair of $\mathrm{L}$ wave $3.5 \mathrm{MHz}$ General Electric A182S-RB transducers, both kinds having a $1 / 2$ in. diameter. Spike-like pulses are applied to the emitter using an Olympus PR5072 pulserreceiver at a $100 \mathrm{~Hz}$ rate. Care has been taken to balance the internal resistances of the signal acquisition channels. Voltage signals are acquired and digitized with a 14-bit resolution and at $f_{s}=400 \mathrm{MHz}$ sampling frequency. The acquisitions are averaged over 1000 iterations.

Absolute determination of the reflection and transmission coefficients $\mathcal{R}$ and $\mathcal{T}$ of the line-slab-line system can be performed by combining the measurements of the reflections by the Dural-air interface of each isolated delay line and the measurement of the reflection and transmission by the lineslab-line system according to the following protocol. With the setup being mounted as sketched in Fig. 1(a) and the transducers being labelled by 1 and 2 , respectively, the signals transmitted by the slab from 1 to $2, S_{t 1}$, and from 2 to 1 , $S_{t 2}$, are acquired. Then, the signals reflected by the sandwiched slab from 1 back to $1, S_{r 1}$, and from 2 back to $2, S_{r 2}$, are acquired. Finally, the line-slab-line system is unmounted, the delay lines are separated, and the sample and the spacer are removed, as sketched in Fig. 1(b). Then, the calibration signals reflected by the delay line ends from 1 toward $1, S_{c 1}$, and from 2 toward 2, $S_{c 2}$, are acquired.

With $I(\omega)$ being the spectrum of the electric signal applied to the emitter ( $\omega=2 \pi f$ is the angular frequency) and $g_{\mathrm{d} m}$ (respectively, $g_{m \mathrm{~d}}$ ) the electro-mechanical transduction coefficient from transducer $m$ to the delay line (resp. from the delay line to transducer $m), m=1,2$, the calibration signal spectra satisfy

$$
S_{c m}(\omega)=-I(\omega) g_{m \mathrm{~d}} g_{\mathrm{d} m} e^{2 j k_{\mathrm{d}} H},
$$

where $k_{\mathrm{d}}=\omega / c^{\mathrm{d}}$ is the wave number in the delay lines. The signals reflected and transmitted by the slab satisfy

$$
\left\{\begin{array}{l}
S_{r m}(\omega)=I(\omega) \mathcal{R} g_{m \mathrm{~d}} g_{\mathrm{d} m} e^{2 j k_{\mathrm{d}} H} \\
S_{t m}(\omega)=I(\omega) \mathcal{T} g_{m \mathrm{~d}} g_{\mathrm{d} n} e^{2 j k_{\mathrm{d}} H}
\end{array}\right.
$$

where $n=2$ if $m=1$ and $n=1$ if $m=2$. Thus, $\mathcal{R}$ and $\mathcal{T}$ can be experimentally evaluated from the following expressions:

$$
\mathcal{R}_{m}=-\frac{S_{r m}}{S_{c m}} \quad \text { and } \quad \mathcal{T}=\sqrt{\frac{S_{t 1} S_{t 2}}{S_{c 1} S_{c 2}}}
$$

where $\mathcal{R}_{1}$ and $\mathcal{R}_{2}$ are two independent evaluations of $\mathcal{R}$ on each side of the setup.

The test material chosen to experimentally validate the technique is water, which is known to behave as a Newtonian fluid at $\mathrm{MHz}$ frequencies. As such, only longitudinal waves can propagate in water. The mechanical properties of water at $5^{\circ} \mathrm{C}$ are mass density $\rho=1000 \mathrm{~kg} \mathrm{~m}^{-3}$, adiabatic sound speed $c_{0}=1426 \mathrm{~m} \mathrm{~s}^{-1}$, shear viscosity $\eta=1.519 \mathrm{mPa} \mathrm{s},{ }^{13}$ and bulk viscosity $\eta_{B}=7 \mathrm{mPa} \mathrm{s} .{ }^{14}$ The complex $\mathrm{L}$ velocity is then given by $c_{\mathrm{L}}=c_{0} \sqrt{1-j \omega\left(\eta_{B}+\frac{4}{3} \eta\right) /\left(\rho c_{0}^{2}\right)} \cdot{ }^{15}$ The reflection and transmission of an $\mathrm{L}$ pulse by a $1 \mathrm{~mm}$ thick layer of pure water are measured. The reflected, transmitted, and calibration raw signals are displayed in Fig. 2. The echoes displayed by the reflected and transmitted signals result from the multiple reflections of the $\mathrm{L}$ pulse inside the water layer combined with its weak attenuation. For spectral analysis, the reflected and calibration signals (respectively, the transmission signal) are ended just before the time of arrival $t_{a}=4 d / c_{\mathrm{L}}^{\mathrm{d}}=37.4 \mu \mathrm{s}$ of the pulse reflected twice on the delay line end (resp. transmitted once through the delay line end and reflected once on it), shown as a dotted line in Fig. 2. This results in a spectral resolution of $\Delta f=t_{a}^{-1} \simeq 30 \mathrm{kHz}$.

As incident pulses are assumed as plane waves in the slab, the slab has to be located in the far-field of the emitter. Moreover, the beam diameter, which is evaluated from its frequency-dependent divergence, has to be smaller than the sample diameter. ${ }^{16}$ This results in the following frequency spans over which signal spectra are valid $0.4-9.3 \mathrm{MHz}$ for $\mathrm{L}$

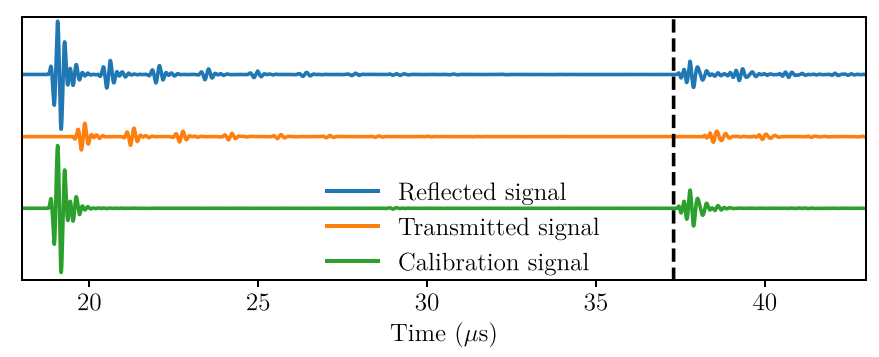

FIG. 2. Signals of reflected and transmitted longitudinal waves by a slab of pure water and calibration signal. Origin of time coincides with the trigger of acoustic emission. 


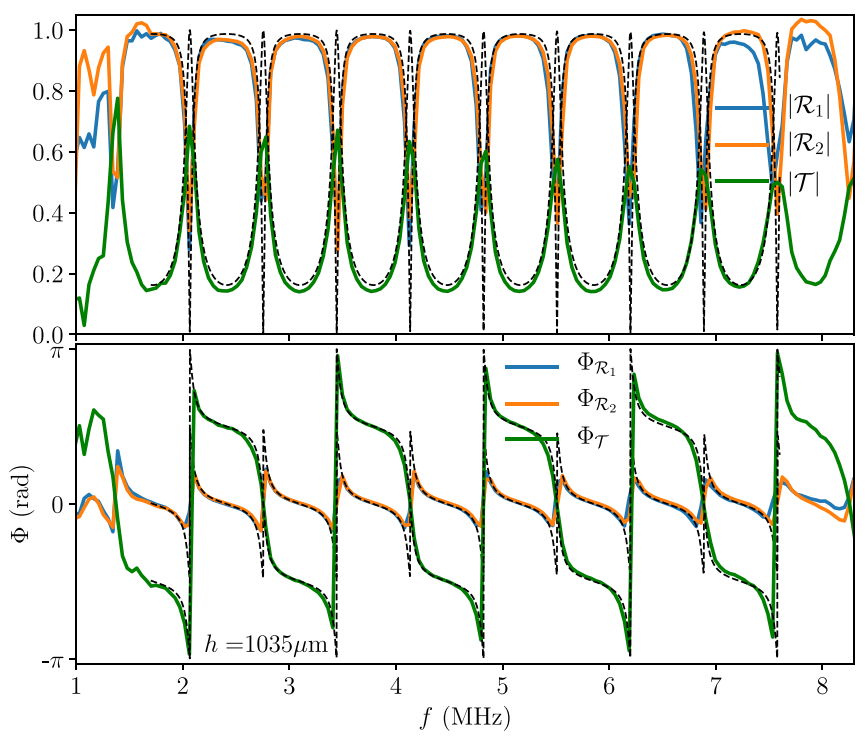

FIG. 3. Amplitude (top) and phase $\Phi$ (bottom) of the reflection and transmission coefficients $\mathcal{R}$ and $\mathcal{T}$ of longitudinal waves by a $1 \mathrm{~mm}$ thick slab of pure water; blue and orange solid curves: reflection coefficients measured on each side of the set-up $\mathcal{R}_{1}, \mathcal{R}_{2}$. Green solid curve: transmission coefficient. Dashed curves: Fabry-Pérot resonator model (1).

waves and $0.2-4.7 \mathrm{MHz}$ for $\mathrm{T}$ waves. Taking also into account the limited transducer bandwidth results in the following frequency spans for rheological measurements $1.7-7.6 \mathrm{MHz}$ for water, $1.4-8 \mathrm{MHz}$ for $\mathrm{L}$ waves, and $0.7-5 \mathrm{MHz}$ for $\mathrm{T}$ waves in couplant.

Figure 3 displays the variation as a function of frequency of the amplitude and phase of $\mathcal{R}$ and $\mathcal{T}$ determined experimentally using (2). Their periodic behavior observed over a broad frequency range of $1.7-7.7 \mathrm{MHz}$ results from multiple interferences inside the slab, the expected value of the period $\operatorname{Re}\left(c_{L}\right) / 2 d=713 \mathrm{kHz}$ being in quantitative agreement with its observed value. The best fit of $\mathcal{R}$ by the FabryPérot resonator model (1) with $h$ as the only free parameter results in $h=1.035 \mathrm{~mm}$, in quantitative agreement with the independent measurement of the spacer thickness. The sharpness of the peaks (anti-peaks) displayed by the prediction of $\mathcal{T}(\mathcal{R})$ is not retrieved by the measurements because of their lack of spectral resolution. This quantitative agreement between these measurements and their theoretical description demonstrates the validity of the technique.

The technique is now used to determine the rheological properties of visco-elastic materials using $\mathrm{T}$ and $\mathrm{L}$ waves. As an illustration of practical interest, here we characterize the Olympus SWC-2 couplant. Its mass density measured using a pycnometer is $\rho=1452 \mathrm{~kg} \mathrm{~m}^{-3}$. It is a hydrosoluble organic couplant and could be sensitive to humidity.

The raw signals reflected and transmitted by a $h=1 \mathrm{~mm}$ thick slab of couplant, shown in Fig. 4(a), do not display a series of echoes but rather a quickly vanishing coda as compared with the short calibration signal, as a consequence of an attenuation larger than for $\mathrm{L}$ waves in water. Measurements have been performed using several different spacers with thickness $h$ ranging from $40 \mu \mathrm{m}$ to $1 \mathrm{~mm}$. As expected, the $h$-dependence of the time of flight of the transmitted signal through the slab, shown in Fig. 4(b), is found to be linear, corresponding to a $\mathrm{T}$ wave velocity $c_{\mathrm{T}}=(1450 \pm 10) \mathrm{m} \mathrm{s}^{-1}$. The resulting approximate value of the impedance $\operatorname{Re}(Z) \simeq 2.1$ MRayl is rather large and close to $Z_{\mathrm{d}}=8.81 \mathrm{MRayl}$, which explains the good performance of this material as a shear wave couplant.

The frequency-dependent amplitude and phase of the experimental reflection and transmission coefficients of $\mathrm{T}$ waves by the slab $\mathcal{R}$ and $\mathcal{T}$ are shown in Fig. 5. As expected, the rather large spacer thickness results in oscillations of $\mathcal{R}$ and $\mathcal{T}$ due to interference phenomena in the slab whose periodicity is related to the material elasticity and amplitude to dissipation in the material. More precisely, the period of the oscillations increases with the real part of the shear modulus $G^{\prime}$ while their amplitude and their decrease with frequency are due to wave attenuation that increases with the imaginary part of the shear modulus $G^{\prime \prime}$.

We apply the same method to the couplant with L waves in order to achieve its full characterization. The frequencydependent amplitude and phase of the experimental reflection and transmission coefficients of $\mathrm{L}$ waves by the slab are shown in Fig. 6.

Given the experimental data, the bulk modulus $K$ is chosen to be modeled using the simplest rheological model of visco-elasticity, namely, the Kelvin-Voigt model

$$
K(\omega)=K_{0}+j \omega \eta_{K}
$$

where $K_{0}$ is the static value of $K$ and $\eta_{K}$ a viscosity which is the signature of microscopic relaxation timescales much smaller than $\omega^{-1}$.

The shear modulus $G$ is found to involve also larger relaxation timescales, of the order of $\omega^{-1}$. Hence, we used a generalized Maxwell model with two relaxation timescales.
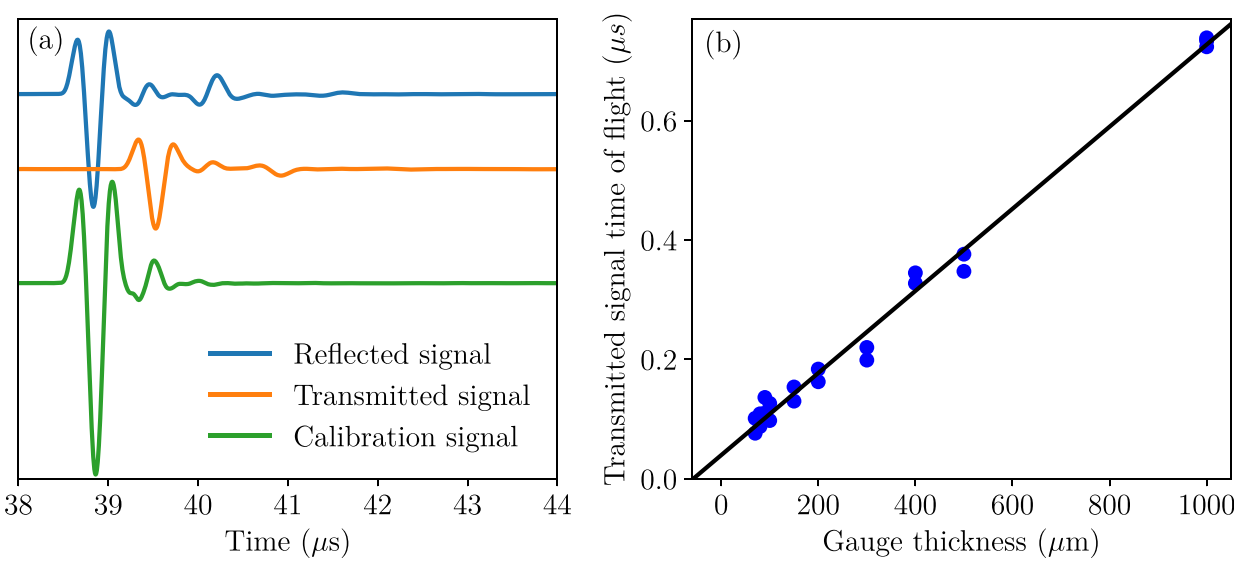

FIG. 4. (a) Shear wave signals reflected and transmitted by a $1 \mathrm{~mm}$ thick slab of Olympus SWC-2 shearwave couplant and calibration signal. Origin of time coincides with the trigger of acoustic emission. (b) Time of flight of the transmitted signal through the slab. Solid line: best linear fit giving the shear wave velocity $\operatorname{Re}\left(c_{\mathrm{T}}\right)$ $=(1450 \pm 10) \mathrm{m} \mathrm{s}^{-1}$. 


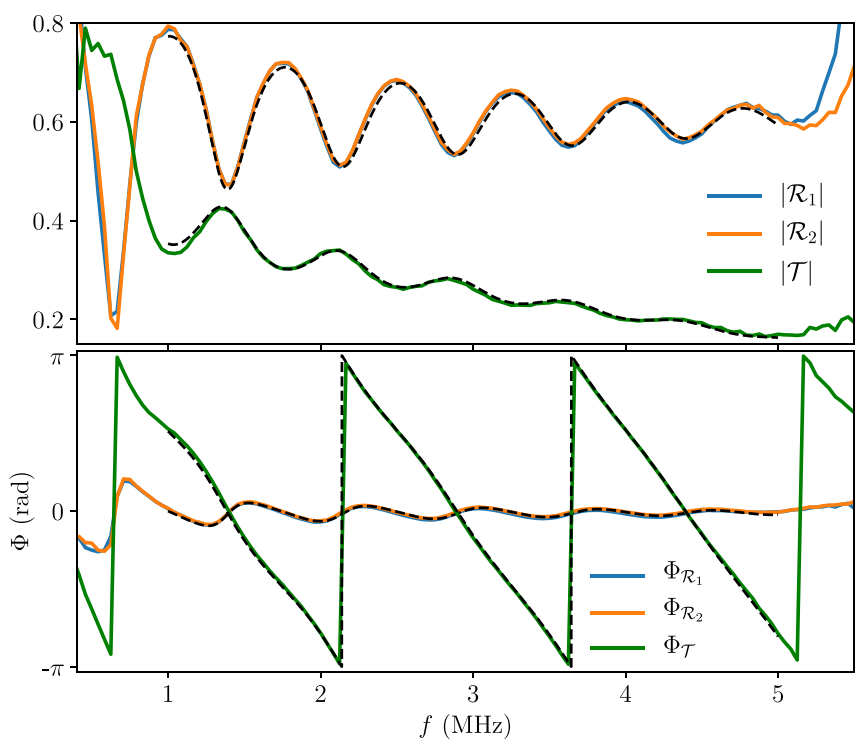

FIG. 5. Amplitude (top) and phase $\Phi$ (bottom) of the reflection and transmission coefficients $\mathcal{R}$ and $\mathcal{T}$ of shear waves by a $1 \mathrm{~mm}$ thick slab of Olympus SWC-2 shear-wave couplant; blue and orange solid curves: reflection coefficients measured on each side of the set-up $\mathcal{R}_{1}, \mathcal{R}_{2}$. Green solid curve: transmission coefficient. Dashed curves: best fit by the Fabry-Pérot resonator model (1) using the visco-elastic model (4) for shear modulus $G$.

With the first one being small compared to $\omega^{-1}$, its contribution is described using the Kelvin-Voigt model. With the second one $\tau$ being comparable with $\omega^{-1}$ in the explored frequency range, its contribution is described using the Zener model. The whole phenomenological model corresponds to a Kelvin-Voigt element in parallel to a Maxwell element or equivalently to a Zener model in parallel to a dashpot

$$
G(\omega)=G_{0}+G_{\infty} \frac{\omega^{2} \tau^{2}+j \omega \tau}{1+\omega^{2} \tau^{2}}+j \omega \eta_{G},
$$

where $G_{0}$ is the static value of $G, G_{\infty}$ an elasticity constant, and $\eta_{G}$ a viscosity. First, we compute the best fit of the real and imaginary parts of both $\mathcal{R}$ and $\mathcal{T}$ for $\mathrm{T}$ waves by (1) using the model (4) for $G$ with $G_{0}, G_{\infty}, \tau$, and $\eta_{G}$ as free parameters. The quantitative agreement between measurements and their best fit by this model, shown in Fig. 5, demonstrates the relevance of the model built to describe $G$. Then, we compute the best fit of the real and imaginary parts of both $\mathcal{R}$ and $\mathcal{T}$ for $\mathrm{L}$ waves by (1) using the expression of $G$ determined formerly and the Kelvin-Voigt model (3) for $K$

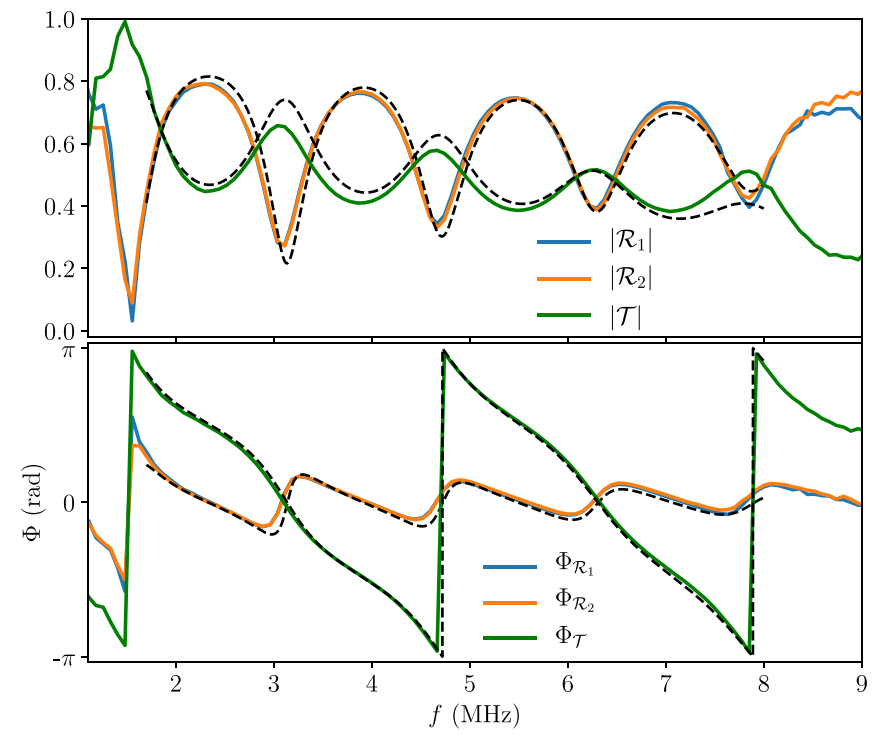

FIG. 6. Amplitude (top) and phase $\Phi$ (bottom) of the reflection and transmission coefficients $\mathcal{R}$ and $\mathcal{T}$ of $\mathrm{L}$ waves by a $1 \mathrm{~mm}$ thick slab of Olympus SWC-2 shear-wave couplant; blue and orange solid curves: reflection coefficients measured on each side of the set-up $\mathcal{R}_{1}, \mathcal{R}_{2}$. Green solid curve: $\mathcal{T}$. Dashed curves: best fit by the Fabry-Pérot resonator model (1) using the hybrid model (4) for shear modulus $G$ and the Kelvin-Voigt visco-elastic model (3) for bulk modulus $K$.

with $K_{0}$ and $\eta_{K}$ as free parameters. The quantitative agreement between measurements and their best fit by the KelvinVoigt model, shown in Fig. 6, demonstrates the relevance of this model to describe $K$ too. Thus, the rheology of the shear-wave couplant has been thoroughly determined using both $\mathrm{L}$ and $\mathrm{T}$ waves.

The corresponding frequency-dependent behavior of $G$ is shown in Fig. 7, evidencing the monotonous increase in $G^{\prime}$ with frequency and the saturation of $G^{\prime \prime}$ at high frequency. The values displayed are based on the averaging of four experiments in the same configuration. This results in the following values: $G_{0}=2.61 \pm 0.09 \mathrm{GPa}, G_{\infty}=0.83 \pm 0.05 \mathrm{GPa}, \tau=121$ $\pm 12 \mathrm{~ns}$, and $\eta_{G}=7.5 \pm 1 \mathrm{~Pa}$. The uncertainties are the standard deviations of those values. For the bulk modulus, the parameters are $K_{0}=10.9 \mathrm{GPa}$ and $\eta_{K}=11.2 \mathrm{~Pa}$ s. The corresponding relaxation timescales can be evaluated as $\eta_{G} / G_{0}$ $\simeq 3 \mathrm{~ns}$ and $\eta_{K} / K_{0} \simeq 1 \mathrm{~ns}$. These timescales are related to the $\mathrm{GHz}$ range and thus well described by the Kelvin-Voigt model in our frequency range. The imaginary part $K^{\prime \prime}$ of the bulk modulus is shown in Fig. 7 for illustration purpose.
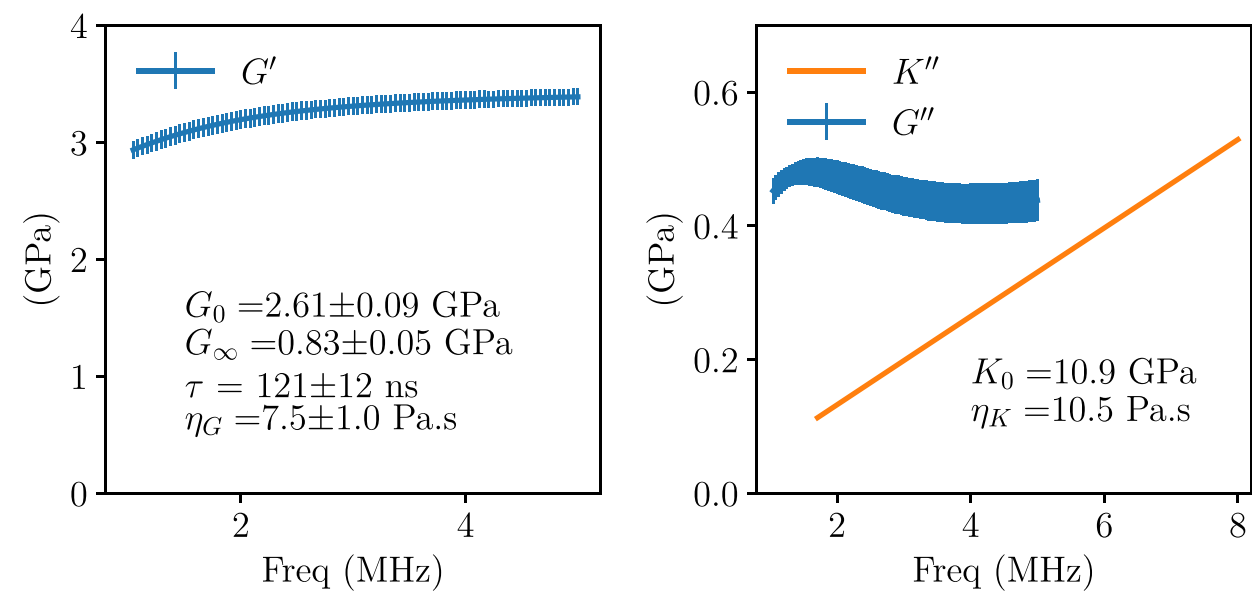

FIG. 7. Frequency dependence of the real part $G^{\prime}$ and imaginary part $G^{\prime \prime}$ of the shear modulus $G$ (left) and imaginary part $K^{\prime \prime}$ of the bulk modulus $K$ (right). These measurements correspond to the properties of the SWC-2 couplant at $(5.0 \pm 0.2)^{\circ} \mathrm{C}$. Error bars are based on statistical variability on four experiments. 
To conclude, we proposed an original ultrasonic technique for measuring the rheological properties of viscoelastic materials. Using Fabry-Pérot resonances displayed by thin samples, highly absorbing materials can be addressed. Complex rheological behavior with relaxation times can be rendered by this high-sensitivity technique.

A direct application of these measurements is the possibility to accurately predict the transmission by a layer of couplant of any thickness at $5^{\circ} \mathrm{C}$. Recently, Treiber et al. proposed a six-step method to get rid of the nonreproducible transmission by tightening-controlled couplant layers in attenuation measurements. ${ }^{17}$ Our work leads us to suggest to control the thickness of the coupling layers in line-slab-line configurations and to take quantitatively account of the effect of the couplant layer when analyzing the reflected or transmitted signals thanks to the knowledge of its rheological properties.

${ }^{1}$ E. P. Papadakis, "Ultrasonic attenuation in thin specimens driven through buffer rods," J. Acoust. Soc. Am. 44, 724-734 (1968).

${ }^{2}$ E. P. Papadakis, "Buffer-rod system for ultrasonic attenuation measurements,” J. Acoust. Soc. Am. 44, 1437-1441 (1968).

${ }^{3}$ E. P. Papadakis, K. A. Fowler, and L. C. Lynnworth, "Ultrasonic attenuation by spectrum analysis of pulses in buffer rods: Method and diffraction corrections," J. Acoust. Soc. Am. 53, 1336-1343 (1973).

${ }^{4}$ R. T. Higuti and J. C. Adamowski, "Ultrasonic densitometer using a multiple reflection technique," IEEE Trans. Ultrason., Ferroelectr., Freq. Control 49, 1260-1268 (2002).
${ }^{5}$ E. Bjorndal, K. E. Froysa, and S. A. Engeseth, "A novel approach to acoustic liquid density measurements using a buffer rod based measuring cell," IEEE Trans. Ultrason., Ferroelectr., Freq. Control 55, 1794-1808 (2008).

${ }^{6}$ S. Hoche, M. A. Hussein, and T. Becker, "Ultrasound-based density determination via buffer rod techniques: A review," J. Sens. Sens. Syst. 2, 103-125 (2013).

${ }^{7}$ N. Samet, P. Maréchal, and H. Duflo, "Ultrasonic characterization of a fluid layer using a broadband transducer," Ultrasonics 52, 427-434 (2012). ${ }^{8}$ N. Ghodhbani, P. Maréchal, and H. Duflo, "Ultrasound monitoring of the cure kinetics of an epoxy resin: Identification, frequency and temperature dependence," Polym. Testing 56, 156-166 (2016).

${ }^{9}$ B. Hartmann and J. Jarzynski, "Immersion apparatus for ultrasonic measurements in polymers," J. Acoust. Soc. Am. 56, 1469-1477 (1974).

${ }^{10} \mathrm{P}$. Longin, C. Verdier, and M. Piau, "Dynamic shear rheology of high molecular weight polydimethylsiloxanes: Comparison of rheometry and ultrasound," J. Non-Newtonian Fluid Mech. 76, 213-232 (1998).

${ }^{11}$ F. Simonetti and P. Cawley, "Ultrasonic interferometry for the measurement of shear velocity and attenuation in viscoelastic solids," J. Acoust. Soc. Am. 115, 157-164 (2004).

${ }^{12}$ L. Brekhovskikh, Waves in Layered Media (Elsevier, 1980).

${ }^{13}$ R. C. Weast and M. J. Astle, CRC Handbook of Chemistry and Physics (CRC, Cleveland, OH, 1969).

${ }^{14}$ X. He, H. Wei, J. Shi, J. Liu, S. Li, W. Chen, and X. Mo, "Experimental measurement of bulk viscosity of water based on stimulated Brillouin scattering," Opt. Commun. 285, 4120-4124 (2012).

${ }^{15}$ L. E. Kinsler, A. R. Frey, A. B. Coppens, and J. V. Sanders, Fundamentals of Acoustics, 4th ed. (John Wiley and Sons, 2009).

${ }^{16}$ D. T. Blackstock, Fundamentals of Physical Acoustics (John Wiley \& Sons, 2000).

${ }^{17}$ M. Treiber, J.-Y. Kim, L. J. Jacobs, and J. Qu, "Correction for partial reflection in ultrasonic attenuation measurements using contact transducers," J. Acoust. Soc. Am. 125, 2946 (2009). 